\title{
Life Cycle Cost Impact of Using Prognostic Health Management (PHM) for Helicopter Avionics
}

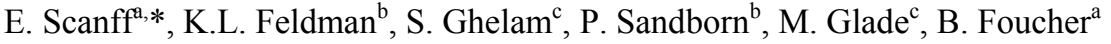 \\ ${ }^{a}$ EADS Corporate Research Center, 12 rue Pasteur, 92150 Suresnes, France \\ ${ }^{\mathrm{b}}$ CALCE EPSC, Department of Mechanical Engineering, University of Maryland, College Park, MD, \\ 20742, USA \\ ${ }^{\circ}$ Eurocopter, Aéroport Marseille Provence, 13725 Marignane Cedex, France \\ *Corresponding author: \\ Tel: 301-405-5329 \\ Fax: 301-314-9269 \\ Email address: escanff@calce.umd.edu
}

\begin{abstract}
Case studies were conducted using a stochastic model to predict the life cycle cost impact associated with the application of Prognostic Health Management (PHM) to helicopter avionics. The life cycle costs of systems that assumed unscheduled maintenance and fixed-interval scheduled maintenance were compared to the costs of precursor-to-failure and life consumption monitoring PHM approaches, and the optimal safety margins and prognostic distances were determined.
\end{abstract}

Key Words - Prognostic Health Management (PHM), life cycle costs prediction, Life Consumption Monitoring (LCM), Health Monitoring (HM), helicopter, avionics.

\section{Introduction}

The aeronautical industry faces major financial challenges and changes that currently motivate cost avoidance measures. Maintenance errors are responsible for $12-15 \%$ of accidents in this sector [1]; while failure to diagnose and correct a problem can be disastrous, overly conservative maintenance scheduling reduces operational availability and prematurely disposes of functioning units thus increasing life cycle costs.

To provide added value for the maintenance process, Prognostic Health Management (PHM) approaches must estimate the remaining life of a Line Replaceable Unit (LRU) in a timeframe suitable for 
corrective action within a distributed maintenance decision-making environment. The estimated annual global market for diagnostic tools within commercial aviation maintenance is in excess of \$1 billion [2], indicating the potential receptiveness to this promising technology.

Previous work has examined the incorporation of prognostics for electronics in military air and ground vehicles, particularly power supplies [3, 4]. These efforts have produced Return-on-Investment (ROI) estimates of the costs of PHM implementation and the potential for cost avoidance, [5, 6]. However, these models quantify neither the reduction in life cycle costs nor the increase in operational reliability or availability gained from PHM and lack the necessary generality to evaluate PHM incorporation for application-specific helicopter avionics. Closer examination of ROI and the implementation of PHM within maintenance scheduling and planning will better support decision making and improve cost avoidance analysis.

The application of Health and Usage Monitoring Systems (HUMS) in helicopters was initially to address safety problems in North Sea helicopters [7]. The use of HUMS has been expanded to facilitate improvements in helicopters maintenance management and to identify where changes in maintenance practices would be beneficial [8]. Eurocopter, a leader in the European helicopter industry, was among the first manufacturers to implement HUMS for rotorcraft and has collected data from over 100,000 flight hours [9]. HUMS utilize a variety of onboard sensors and data acquisition systems and can process data onboard, on ground stations, or a combination of the two; HUMS enables the comparison of accumulated data against accepted performance criteria and the issuance of maintenance indicators [10].

This study is part of an effort by Eurocopter to reduce maintenance-related costs and improve aircraft operability ${ }^{1}$ through the use of an integrated PHM approach. Eurocopter's cost avoidance estimate associated with incorporating PHM into its maintenance procedure was conducted using the PHM maintenance planning model developed by the Center for Advanced Life Cycle Engineering (CALCE) at the University of Maryland [12]. Business cases were constructed using Eurocopter's data from manufacturers of two standard microelectronic subsystems in commercial helicopters, a Processing Unit (PU) and a Multi-Function Display (MFD), Figures 1 and 2.

\footnotetext{
${ }^{1}$ Operability is an aggregate measure representing a balance between availability and operational reliability, [11].
} 


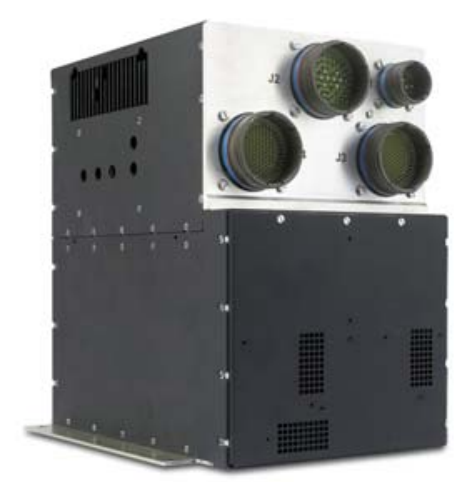

Figure 1- Processing Unit (PU)

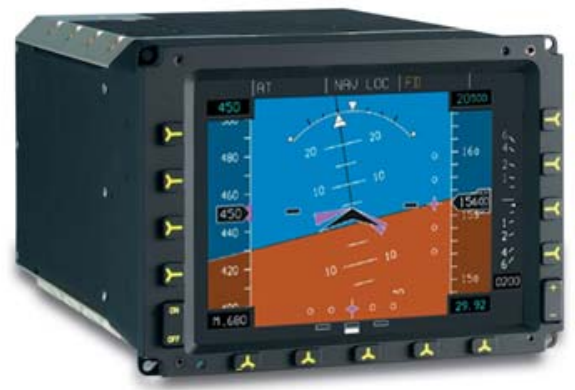

Figure 2- Multi-Function Display Unit (MFU)

\section{Model description}

CALCE's PHM maintenance planning software, [12], enables an assessment of the costs and benefits of implementing PHM and was used to model the costs associated with Health Monitoring (HM) and Life Consumption Monitoring (LCM) approaches. HM (precursor-to-failure), is modeled as a LRU-dependent approach because the monitoring structure ${ }^{2}$ is assumed to be coupled to the specific LRU's variations in manufacturing or materials, and thus the reliability of a particular LRU instance. LCM is an LRUindependent approach, which is assumed to be uncoupled from a specific LRU's variations in manufacturing or materials and the monitoring structure predicts failure based on the expected reliability of a nominal LRU. These approaches represent alternatives to the unscheduled and fixed scheduled maintenance interval methods currently in practice.

\footnotetext{
2 "Monitoring structure" refers to the system attribute that is monitored or the PHM-specific components/sensors added to the system that serve as monitors and/or fuses.
} 
The inputs to the model are the probability distributions associated with time to failure of the LRUs, the probability distributions associated with the PHM approaches' ability to indicated impeding failure, and parameters describing the scheduled or unscheduled maintenance activities (e.g., Table 1).

In the model, discrete-event simulation is used to follow the life of individual socket ${ }^{3}$ instances from the start of their field life to the end of their operation and support. The model used in this paper treats all inputs to the discrete-event simulation as probability distributions, i.e., a stochastic analysis is used, which is implemented as a Monte Carlo simulation. Various maintenance interval and PHM approaches are distinguished by how sampled TTF values are used to model PHM forecasting distributions. For example, in the case of precursor to failure (an LRU-dependent PHM approach), for each LRU time to failure (TTF) distribution sample, a precursor to failure monitoring TTF distribution is created that is centered on the LRU TTF minus the prognostic distance. ${ }^{4}$ The precursor to failure monitoring TTF distribution is then sampled and if the precursor to failure monitoring TTF sample is less than the actual TTF of the LRU instance then precursor to failure monitoring was successful and a "scheduled" maintenance activity is performed resulting in the timeline for the socket being incremented by the precursor to failure monitoring sampled TTF. If the precursor to failure monitoring distribution TTF sample is greater than the actual TTF of the LRU instance then precursor to failure monitoring was unsuccessful and an "unscheduled" maintenance activity is performed resulting in the timeline for the socket being incremented by the actual TTF of the LRU instance. For each type of maintenance activity, the relevant costs are accumulated. See [11] for a detailed description of the models for other PHM approaches.

The model provides the ability to determine and optimize the prognostic distance or safety margin associated with the PHM approach (i.e., to find the prognostic distance or safety margin that minimizes the life cycle cost of the socket). The prognostic distance or safety margin is a variable of interest for maintenance planners and cost modelers.

Two TTF distribution shapes were considered in the analysis: an exponential and a Weibull distribution (Figures 3 and 4). Initial observations of the two LRUs shown in Figures 1 and 2 indicate that

\footnotetext{
3 A "socket" is the location of a LRU. For example, a socket could be a location on an engine that is occupied by an engine controller (the LRU). During the life of the engine, if the engine controller fails and is replaced, the socket will be occupied by several LRUs. The model used in this study follows the lifetime cost of "sockets", not LRUs.

4 The prognostic distance is a measure of how long before system failure the prognostic structures or prognostic cell is expected to indicate failure (in operational hours for example), i.e., the interval between an indication from the monitoring structure and the actual failure of the LRU itself. In the case of LRU-independent PHM approaches, the interval is called the safety margin.
} 
their failure rate is approximately constant, which is associated with an exponential TTF distribution. However, failure mechanisms associated with new technologies, as highlighted in recent papers $[13,14]$, suggest that Weibull distributions, which have been used extensively in failure predictions for many aeronautical applications [15], may prove to be more representative for future LRUs. The Weibull distributions depicted in Figures 3 and 4 were obtained under the assumption that the Weibull distribution approximates a normal distribution centered on the MTBF value with a standard deviation of 100 hours.

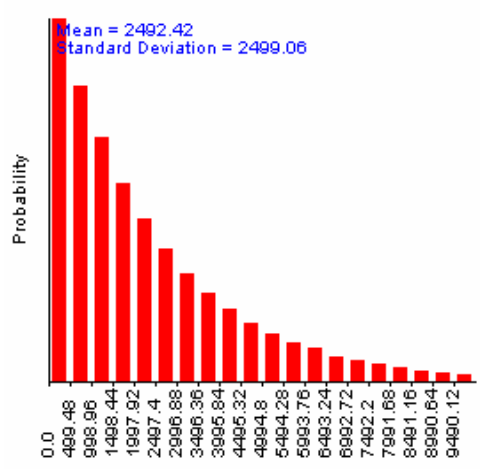

Time to F ailure

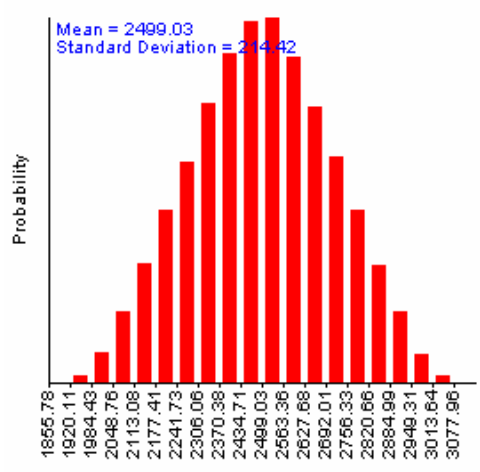

Time to Failure

\begin{tabular}{|c|c|c|}
\cline { 2 - 3 } \multicolumn{1}{c|}{} & Exponential distribution $^{5}$ & Weibull distribution \\
\hline$\gamma$ (location parameter) & 0 & 2320 \\
\hline$\beta$ (shape parameter) & 1 & 3 \\
\hline $\begin{array}{c}\text { (see underneath values } \\
\text { for description) }\end{array}$ & $\Lambda$ (rate parameter, exponential) & $\eta$ (scale parameter, Weibull) \\
\hline
\end{tabular}

Figure 3- Processing Unit time to failure distributions shape and parameters

a) Exponential - left

b) Weibull - right
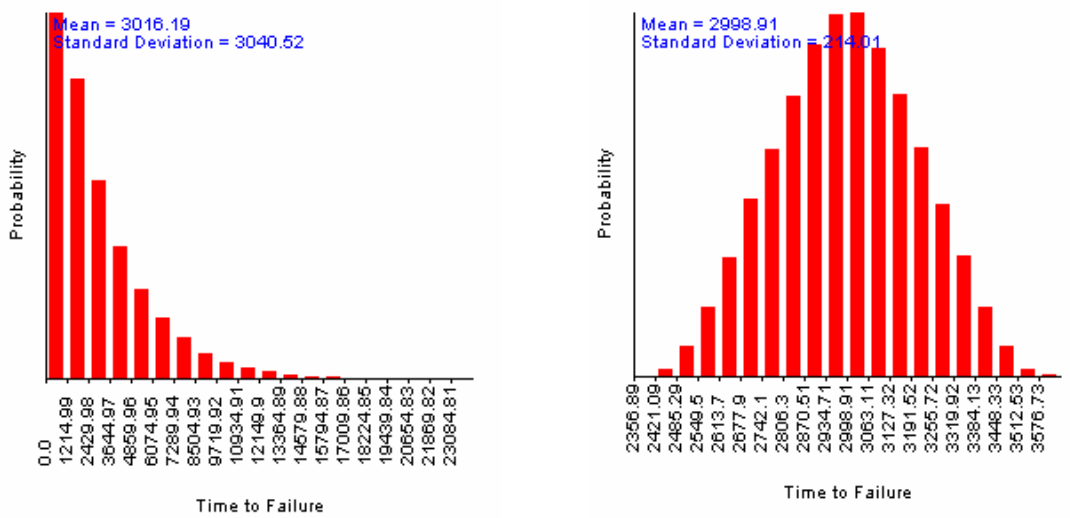

\begin{tabular}{|c|c|c|}
\hline & Exponential distribution & Weibull distribution \\
\hline$\gamma$ (location parameter) & 0 & 2820 \\
\hline
\end{tabular}

\footnotetext{
${ }^{5}$ The exponential distribution is a special case of the Weibull distribution, where $\beta=1$. The location parameter $\gamma$ shifts the beginning of the distribution.
} 


\begin{tabular}{|c|c|c|}
\hline$\beta$ (shape parameter) & 1 & 3 \\
\hline $\begin{array}{c}\text { (see underneath values } \\
\text { for description) }\end{array}$ & $\Lambda$ (rate parameter, exponential) & $\eta$ (scale parameter, Weibull) \\
\hline
\end{tabular}

Figure 4- Multi-Functional Display Unit time to failure distributions shape and parameters

a) Exponential - left

b) Weibull - right

The discrete-event simulation was used to predict the cost for 100,000 sockets for which the LRUs that occupy the sockets have the TTF distributions shown in Figure 3 and 4. The input data for each piece of equipment are summarized in Table 1. The MTBFs were 2500 and 3000 operational hours and both were subjected to 300 operational hours per year with a minimum field life of 15 years. The unscheduled and scheduled value of each hour out of service, the time to repair, time to replace, the cost of repair, and fraction of repairs requiring replacement of the LRU were inputs to the model. The mean life cycle costs were calculated and the unscheduled, scheduled, and PHM maintenance approaches were examined. The outputs provided by the simulation are life cycle cost, failures avoided, and operational availability.

Table 1- Example operational description and maintenance model input data

\begin{tabular}{|c|c|c|c|c|}
\hline & \multicolumn{2}{|c|}{ Processing Unit (PU) } & \multicolumn{2}{|c|}{$\begin{array}{l}\text { Multi-Function Display } \\
\text { (MFD) }\end{array}$} \\
\hline Production cost ( $€$ per unit) & \multicolumn{2}{|c|}{$\mathrm{x}^{*}$} & \multicolumn{2}{|c|}{$y^{*}$} \\
\hline Time to failure (hours) - MTBF & \multicolumn{2}{|c|}{2500} & \multicolumn{2}{|c|}{3000} \\
\hline Operational hours per year & \multicolumn{2}{|c|}{300} & \multicolumn{2}{|c|}{300} \\
\hline \multirow[t]{2}{*}{ Sustainment life } & \multicolumn{2}{|c|}{15} & \multicolumn{2}{|c|}{15} \\
\hline & Unscheduled & Scheduled & Unscheduled & Scheduled \\
\hline Value of each hour out of service & 3995 & 3000 & 7555 & 4200 \\
\hline Time to repair (hours) & 11 & 8.5 & 14 & 12.5 \\
\hline Time to replace (hours) & 3 & 2.5 & 3 & 2.5 \\
\hline Cost of repair (€) & 1100 & 826 & 1932 & 1074 \\
\hline Fraction of repair requiring replacement & 0.7 & 0.7 & 0.8 & 0.8 \\
\hline \multicolumn{5}{|l|}{ Random failure rate (\%/year) } \\
\hline $1 / \mathrm{MTBF}^{*} 100 * 300$ & \multicolumn{2}{|c|}{12} & \multicolumn{2}{|c|}{10} \\
\hline $1 / 2 \mathrm{MTBF}^{*} 100 * 300$ & \multicolumn{2}{|c|}{6} & \multicolumn{2}{|c|}{5} \\
\hline $1 / 4 \mathrm{MTBF}^{*} 100 * 300$ & \multicolumn{2}{|c|}{3} & \multicolumn{2}{|c|}{2.5} \\
\hline
\end{tabular}

* The production costs have been removed for confidentiality reasons.

\section{Results}

\subsection{Fixed interval scheduled maintenance and unscheduled maintenance}

The unscheduled and fixed interval scheduled maintenance variation for the Weibull and exponential distributions are depicted in Figure 5 for the processing unit. As the fixed scheduled maintenance interval increases, the life cycle costs approach the cost of unscheduled maintenance. Indeed, for a large fixed 
scheduled maintenance interval, virtually every instance of an LRU in a socket will fail before the scheduled maintenance occurs. However, for short maintenance intervals, the cost can become significant because, while expensive unscheduled maintenance actions are avoided, the life cycle cost per unit is high because a large amount of remaining life in the LRUs is thrown away. An optimum maintenance interval to minimize the life cycle cost may exist between these two extreme situations. The curve for the Weibull distribution has a "step" in it due to fluctuations in the number of LRUs needed by the socket. For example, when the fixed maintenance interval is 2000 operational hours, nearly every socket needs two LRUs to satisfy its operational life. When the fixed interval is increased to 2500 operational hours, some sockets will only need one LRU to satisfy their lives, thus decreasing the effective life cycle cost of the socket. The same "step" effect exists for the exponential TTF distribution, but is much less pronounced because the number of LRUs needed per socket is larger.

For the Weibull TTF distribution, the scheduled maintenance can be made more cost-effective by selection of the maintenance interval, compared to the unscheduled maintenance. In the case of an exponential distribution, we found that the unscheduled maintenance is more cost-effective than any fixedinterval scheduled maintenance. The TTF distribution shape is a relevant parameter that influences the choice of maintenance approach.

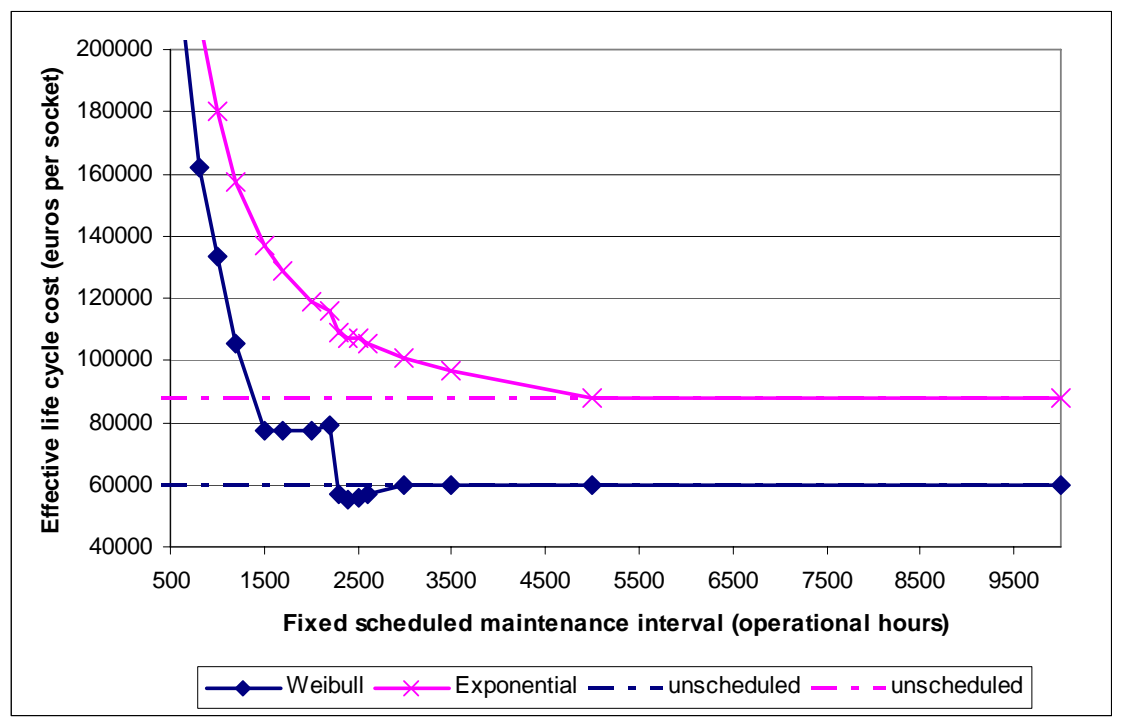

Figure 5-Comparison of the life cycle costs for the Weibull and exponential distributions for the processing unit. 
The unscheduled and scheduled maintenance approaches are well known and understood. The focus will now be on the PHM maintenance approaches in order to assess their usefulness compared to the traditional maintenance methods.

\subsection{PHM maintenance}

The analysis method discussed in Section 2 (and [12]) differentiates between two PHM sustainment methods, PHM LRU-dependent and PHM LRU-independent maintenance.

\subsubsection{PHM LRU-dependent maintenance (Health Monitoring)}

The PHM LRU-dependent model (HM - Health Monitoring) is defined as a fuse or other monitored structure that is manufactured with or within the LRU. The shape and the width of the HM distribution represent the probability of the monitored structure indicating the precursor to a failure at a specific time relative to the actual failure time. The parameter to be optimized in the LRU-dependent (HM) case is the prognostic distance, which is a measure of how long before system failure the monitored structure is expected to indicate failure. In the case of LRU-dependent PHM, the assumption is that the monitored structure will be designed to fail (or indicate failure) a prognostic distance before the actual LRU failure and that the LRUs will be replaced upon failure indication from the monitored structure. ${ }^{6}$ The effective life cycle cost for the Weibull distribution is plotted in Figure 6 as a function of the prognostic distance and the width of the distribution associated with the probability of the monitored (HM) structure indicating failure. First, the increase in the HM distribution width leads to a smoothing of the curve and the minimum costs are higher than those with narrower distributions (which is intuitive). Note, the results indicate that the zero width distribution corresponds to the lowest life cycle cost. This result is also intuitive, it assumes that the HM approach can perfectly indicate failure and therefore no prognostic distance is needed. While this result is not realistic, this case provides important insight into the impact of HM distribution width on life cycle cost optimization.

\footnotetext{
${ }^{6}$ Note, in this model, the optimum prognostic distance (or safety margin for LRU-independent PHM) specifies the replacement time replacement of the LRU earlier or later will cost more on average. The prognostic distance is not a buffer for planning maintenance; it is a buffer for mitigating the uncertainties in the accuracy of the PHM approach.
} 


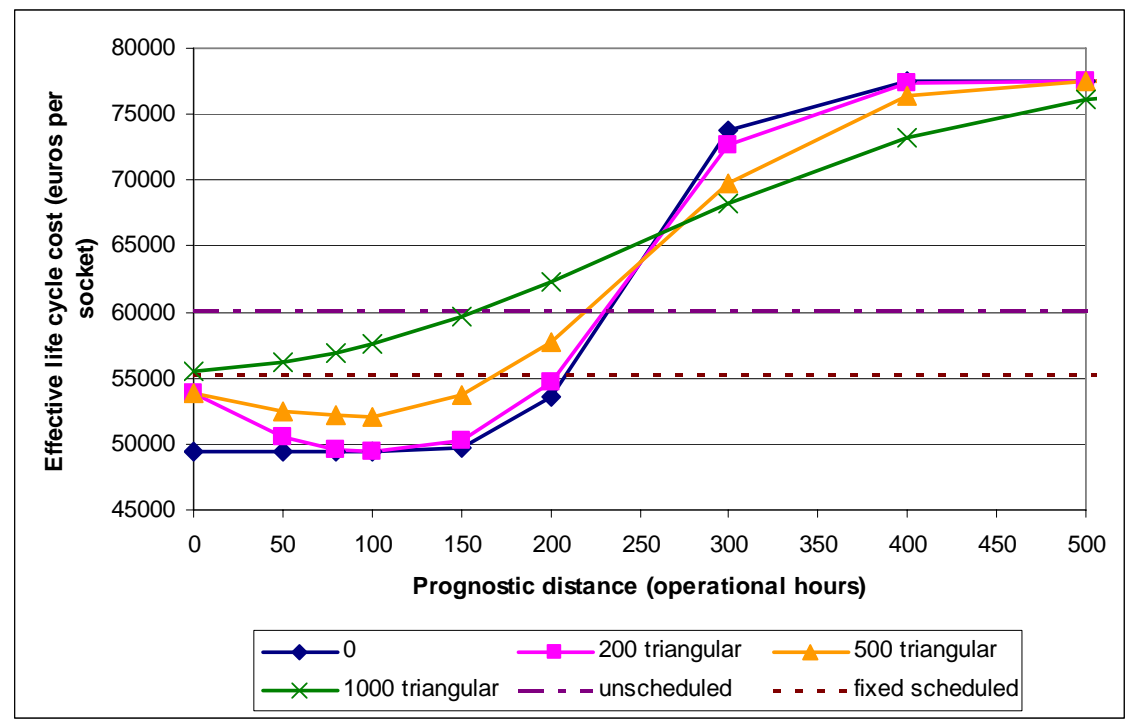

Figure 6- Weibull distribution for the processing unit.

Variation of the effective life cycle cost with the prognostic distance for various triangular distributions representing the HM approach's probability of indicating a failure. The triangular distribution was selected to be illustrative of an arbitrary case. The quantities that differentiate the curves are the width of the HM triangular distribution in operational hours.

No failure outside the scope of the PHM assumed.

The minimum life cycle cost increases with the HM distribution width due to the fact that the PHM approach's ability to detect failure early enough to perform scheduled maintenance is decreasing. For 200- and 500-hour HM distribution widths, the optimum prognostic distance is approximately 100 operational hours. For larger prognostic distances, the costs increase because the prognostic structure indicates the failure too early and valuable life is thrown away. The same observations can be made for the TTF exponential distribution (Figure 7). The PHM LRU-dependent approach will then be cost-effective if defined by adequate features (prognostic distance and PHM width). If we assume here that a 200-hour distribution width PHM structure is realistic, then the life cycle cost is minimized for a prognostic distance of 100 hours at $49,486 €$ for the Weibull distribution and $75,237 €$ for the exponential distribution. One particular case that needs to be explained is the 1000-hour width HM distribution shown in Figure 6. When the HM distribution becomes wide enough, the solution starts to prefer unscheduled maintenance, i.e., unless the PHM approach can catch an error right before failure, the solution would rather do unscheduled maintenance. As a result, the solution sets the prognostic distance to a negative number (allowing it to occasionally catch a failure, but more importantly, also allowing one less LRU to occupy the socket in the 
socket's life). Note, this answer is not wrong, but it is also probably not practical once the cost of implementing the HM solution is incorporated into the analysis.

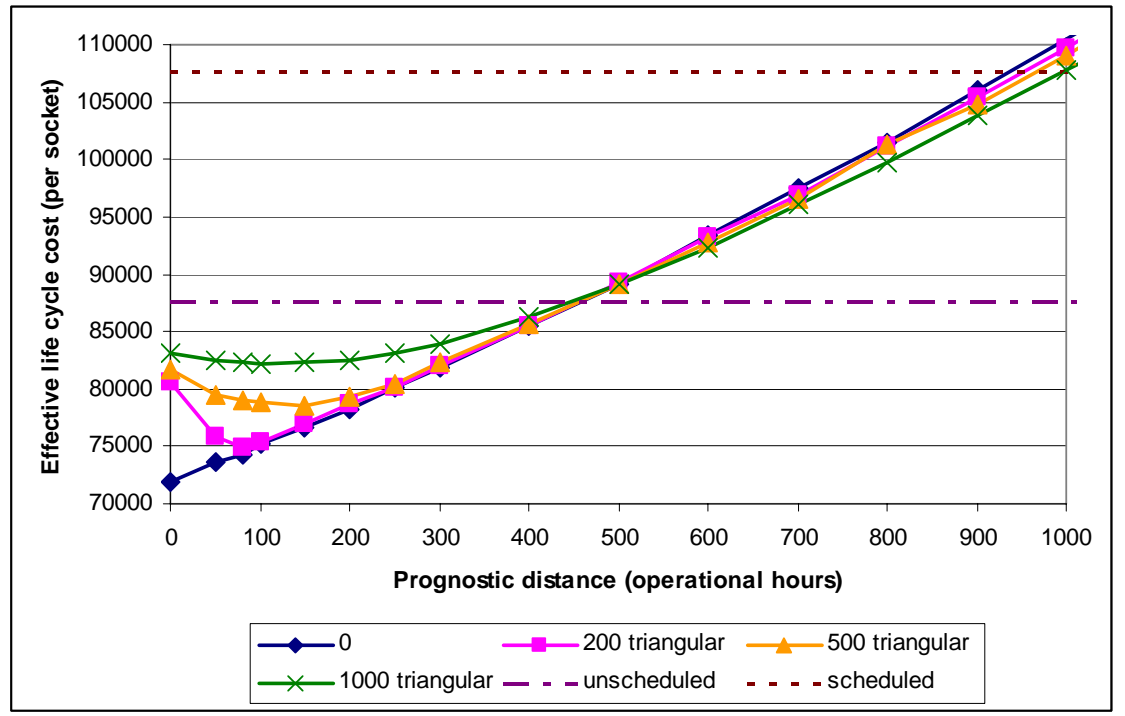

Figure 7- Exponential distribution for the processing unit.

Variation of the effective life cycle cost with the prognostic distance for various triangular distributions representing the HM approach's probability of indicating a failure. The triangular distribution was selected to be illustrative of an arbitrary case. The quantities that differentiate the curves are the width of the HM triangular distribution in operational hours.

No failure outside the scope of the PHM assumed.

\subsubsection{PHM LRU-independent maintenance (Life Consumption Monitoring)}

The other PHM approach treated here is the PHM LRU-independent method. The PHM approach is independent of the specific instance of the LRU, and its failure predictions are based on a nominal (expected) version of the LRU. An example is the Life Consumption Monitoring (LCM), which uses the environmental stress history seen by a particular LRU instance and a model of the nominal LRU to predict remaining life, [16]. The parameter to be optimized here is the safety margin, which is the length of time before the LRU-independent method indicated failure. In the case of the LRU-independent PHM, the assumption is that the LRUs will be replaced at a time equal to the predicted LRU failure less the safety margin. Using this approach with an exponential TTF distribution, predicts large life cycle costs, and the PHM LRU-independent approach is not cost-beneficial compared to the current sustainment approaches (scheduled and unscheduled maintenances). However, this is not the behavior we observed with a Weibull distribution (Figure 8). The plot's trends are approximately equivalent to those of the PHM LRU-dependent method: the minimum life cycle cost increases as the width of the PHM distribution increases for safety 
margins less than 250 operational hours. For larger safety margins, the cost increases due to PHM-indicated maintenance taking place too early, representing a tradeoff between frequency of maintenance and unscheduled maintenance. The PHM LRU-independent approach was cost-effective for a 200 -hour width distribution, and a 100-hour safety margin, the resulting life cycle cost was 50,197€.

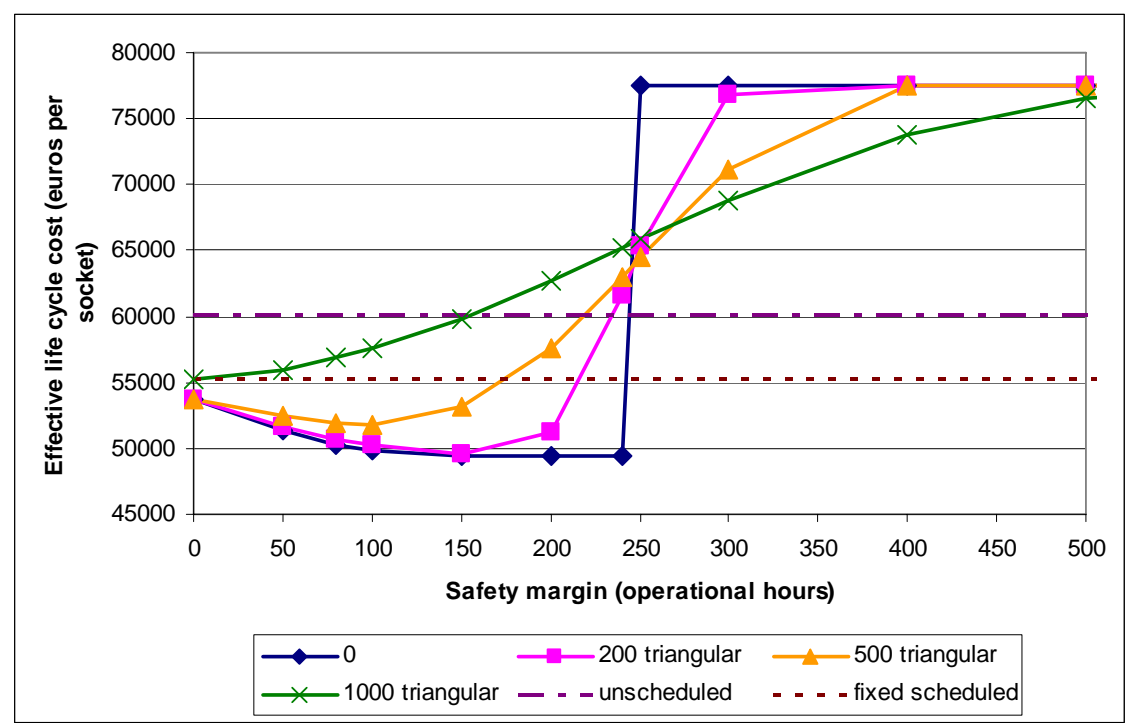

Figure 8- Weibull distribution for the processing unit.

Variation of the effective life cycle cost with the safety margin for various triangular distributions representing the LCM approach's probability of indicating a failure. The quantities that differentiate the curves are the width of the LCM triangular distribution in operational hours.

No failure outside the scope of the PHM assumed.

\subsection{Discussion}

In the results presented in Figures 5-8, the random failure rates (failures due to non-PHM detected events that are outside the scope of the PHM approach used) were considered negligible in order to simplify calculations. The calculations with non-zero random failure rates were performed and the results are summarized in Tables 2 and 3 for the processing and multi-functional display units.

In the tables, results are given for the optimum conditions in each maintenance approach. Thus, 2400 and 2900 operation hours are selected as the fixed scheduled maintenance interval respectively for the processing units and the multi-function display. For both units, a 200-hour triangular PHM distribution width was selected for the PHM approaches with prognostic distance or safety margin of 100 operational hours.

Table 2 - Life cycle costs for the processing unit (euros per socket)

(RF: Random Failure rate) 
Microelectronics Reliability, Vol. 47, No. 12, pp. 1857-1864, December 2007

\begin{tabular}{|c|c|c|c|c|c|c|c|c|}
\hline $\begin{array}{c}\mathbf{R F} \\
\mathbf{\%} / \mathbf{y r})\end{array}$ & $\begin{array}{c}\text { Un- } \\
\text { scheduled }\end{array}$ & $\begin{array}{c}\text { Fixed } \\
\text { Interval }\end{array}$ & $\begin{array}{c}\text { PHM } \\
\text { Dependent }\end{array}$ & $\begin{array}{c}\text { PHM } \\
\text { Indepen- } \\
\text { dent }\end{array}$ & $\begin{array}{c}\text { Un- } \\
\text { scheduled }\end{array}$ & $\begin{array}{c}\text { Fixed } \\
\text { Interval }\end{array}$ & $\begin{array}{c}\text { PHM } \\
\text { Dependent }\end{array}$ & $\begin{array}{c}\text { PHM } \\
\text { Indepen- } \\
\text { dent }\end{array}$ \\
\hline $\mathbf{0}$ & 88,035 & 107,436 & 75,237 & $3,859,793$ & 60,105 & 55,237 & 49,486 & 50,197 \\
\hline $\mathbf{3}$ & 107,066 & 123,214 & 94,920 & $3,873,935$ & 74,855 & 71,184 & 66,326 & 67,157 \\
\hline $\mathbf{6}$ & 132,919 & 141,694 & 120,881 & $3,886,680$ & 92,426 & 89,963 & 85,875 & 87,048 \\
\hline $\mathbf{1 2}$ & 194,150 & 194,327 & 184,368 & $3,914,458$ & 142,735 & 141,735 & 141,341 & 141,247 \\
\hline
\end{tabular}

Table 3 - Life cycles costs for the multi-function display (euros per socket) (RF: Random Failure rate)

\begin{tabular}{|c|c|c|c|c|c|c|c|c|}
\cline { 2 - 9 } \multicolumn{1}{c|}{} & \multicolumn{3}{|c|}{ Exponential Distribution } & \multicolumn{3}{c|}{ Weibull Distribution } \\
\hline $\begin{array}{c}\mathbf{R F} \\
\mathbf{( \% / y r )}\end{array}$ & $\begin{array}{c}\text { Un- } \\
\text { scheduled }\end{array}$ & $\begin{array}{c}\text { Fixed } \\
\text { Interval }\end{array}$ & $\begin{array}{c}\text { PHM } \\
\text { Dependent }\end{array}$ & $\begin{array}{c}\text { PHM } \\
\text { Indepen- } \\
\text { dent }\end{array}$ & $\begin{array}{c}\text { Un- } \\
\text { scheduled }\end{array}$ & $\begin{array}{c}\text { Fixed } \\
\text { Interval }\end{array}$ & $\begin{array}{c}\text { PHM } \\
\text { Dependent }\end{array}$ & $\begin{array}{c}\text { PHM } \\
\text { Indepen- } \\
\text { dent }\end{array}$ \\
\hline $\mathbf{0}$ & 130,887 & 157,535 & 104,678 & $6,194,563$ & 98,217 & 84,453 & 77,659 & 79,458 \\
\hline $\mathbf{2 . 5}$ & 159,163 & 180,187 & 133,519 & $6,216,858$ & 115,963 & 105,534 & 99,177 & 100,841 \\
\hline $\mathbf{5}$ & 195,161 & 207,677 & 170,155 & $6,237,560$ & 137,575 & 130,813 & 125,195 & 126,458 \\
\hline $\mathbf{1 0}$ & 286,326 & 285,755 & 262,197 & $6,274,039$ & 207,817 & 206,674 & 206,198 & 206,457 \\
\hline
\end{tabular}

The two pieces of equipment considered in this study have different functions, production costs, and TTFs. As a consequence, the life cycle costs for each maintenance approach are different, yet the same conclusions can be made for the components with regards the TTF distribution. Increasing the random failure rates increased the costs, as expected, and smoothed the curves. Moreover, one can observe that the PHM approaches provide little benefit when the random failure rate (i.e., failures outside the scope of the PHM approach) exceeds 10\%. It can be inferred that PHM approaches would not be suited for applications with high aleatory uncertainty. In both distribution cases, the PHM-dependent (Health Monitoring) maintenance approach is found to be cost effective compared to the traditional approaches. The PHMindependent approach (e.g., Life Consumption Monitoring) is cost-effective when the TTF is represented as a Weibull (or normal) distribution.

The costs related to unscheduled maintenance are not very different from the costs related to fixed scheduled maintenance in Table 1. An unscheduled maintenance usually carries a high penalty, either in unavailability of spares or qualified technicians, or in cost of excess personnel and spares. The relative benefit of every maintenance approach compared to the fixed interval scheduled maintenance versus the percentage of Random Failure rate is plotted in Figure 9, for the case of the processing unit with Weibull TTF distribution. The unscheduled maintenance is not beneficial when the random failure rate is less than $12 \%$, whereas the PHM approaches enables a maximum benefit of $10 \%$ compared to the life cycle costs calculated for the fixed interval scheduled maintenance. Higher unscheduled maintenance costs (twice as 
large as those given in Table 1, i.e., 2:1) result in a higher benefit (22\%) from the PHM approaches compared to the unscheduled and fixed scheduled maintenance approaches.

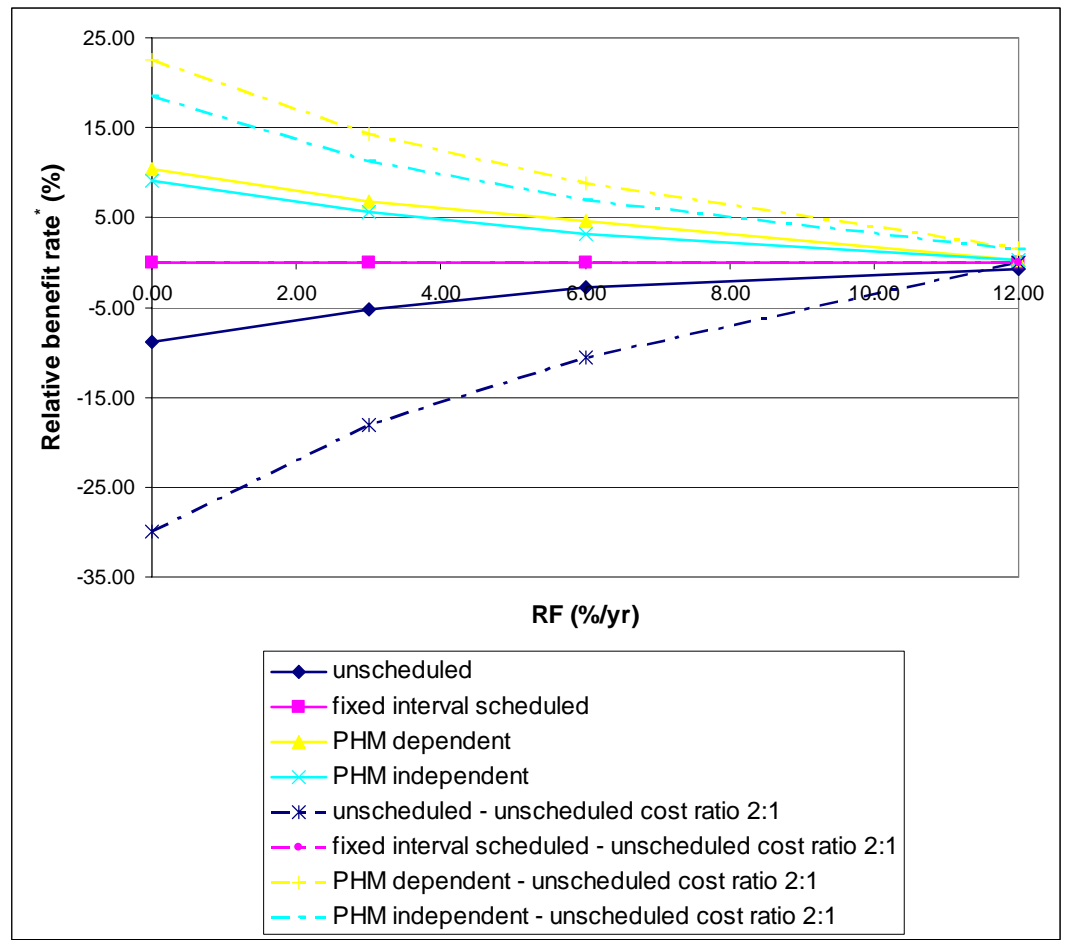

Figure 9- Processing unit - Weibull TTF distribution.

Relative benefit rate ( $\mathrm{RBr}$ ) of the PHM approaches compared to the fixed interval scheduled maintenance.

$$
R B r=\frac{F S-x}{F S}
$$

FS: fixed interval scheduled maintenance life cycle cost $\mathrm{x}$ : selected maintenance life cycle cost

The same conclusions can be made with the exponential distribution and for the multi-functional display unit.

\section{Conclusion}

This paper presents a life cycle cost calculation of PHM for helicopter avionics equipment. The positive impact of prognostic technologies on maintenance costs is primarily the decrease of downtimes and extra costs due to unscheduled maintenance. The model demonstrates the decrease of unscheduled maintenance operations and deduces the associated benefits. The model also enables decision-making concerning PHM tools on a financial basis and enables quantification of the ROI of PHM approaches. The study determined prognostic distances and safety margins associated with a PHM distribution width that 
would optimize the life cycle cost and can be used to determine whether to implement PHM or traditional maintenance approaches.

Results indicate that the precursor-to-failure PHM methods (health monitoring) are superior to LRUindependent methods provided that the precursor method's prognostic distances are smaller than the safety margins for the LRU-independent approach and that equivalent constraints apply ${ }^{7}$. For the LRU-dependent method, results are not significantly affected by the width of the TTF distribution; conversely, the LRUindependent method's life cycle costs vary with the width of the distribution. The prognostic distance narrows with the TTF distribution as variability declines. Thorough knowledge of the TTF distribution shape for a specific microelectronic unit is necessary for the optimal interpretation of PHM life cycle costs.

It is interesting to note that LRU-independent methods become cost effective for systems where time to failures are represented by a Weibull distribution as opposed to those whose time to failure is represented by an exponential distribution. It was also observed that the PHM approaches provide little benefit when the random failure rate exceeds $10 \%$ (random failures are those that are outside the scope of the PHM approach).

The results obtained depend on the assumptions made in this study and are specific to this study. The current model does not consider hybrid prognostics based on LCM and LRU-independent methods. The consequences of random equipment failures cannot be mitigated by PHM approaches. The non-recurring costs of acquiring the prognostic technologies have not been evaluated. Conditions particularly relevant to aircraft, such as the presence of redundant systems and the failure of repair actions to restore avionics to their original conditions, present additional areas for development. The model used has yet to adapt the PHM approach to the operational tempo of maintenance servicing for commercial air traffic as determined by airline or offshore operator policies and mandated by regulatory bodies [17]. While this study concerns PHM approaches as applied to microelectronic equipment, it has implications within the global health management framework at the aircraft level.

\section{Acknowledgements}

\footnotetext{
${ }^{7}$ The result is contingent upon the applicability of the LRU-dependent (precursor to failure) type of PHM approach for helicopter avionics.
} 
Microelectronics Reliability, Vol. 47, No. 12, pp. 1857-1864, December 2007

This work has been supported by the EADS group via its membership to the CALCE Consortium. The authors also wish to thank P. Asseman from Airbus and F. Viniacourt from EADS CCR for their valuable discussions and comments on the paper.

\section{References}

[1] M.S. Patankar, J.C. Taylor, "Risk management and error reduction in aviation maintenance," Ashgate; 2003.

[2] R. Wylie, R. Orchard, M. Halasz, F. Dubé, "IDS: improving aircraft fleet maintenance," Proceedings of the 14th National Conference on Artificial Intelligence and Innovative Applications of AI (IAAI-97), Providence, RI; July 27-31, 1997.

[3] D.L. Goodman, S. Wood, A. Turner, "Return on investment (ROI) for electronic prognostics in mil/aero systems," Proceedings IEEE AutoTest Conference, Orlando, FL; September 2005, p. 73-75.

[4] J. Banks, K. Reichard, E. Crow, K. Nickell, "How engineers can conduct cost-benefit analysis for PHM systems,” Proceedings IEEE Aerospace Conference, Big Sky, MT; March 2005, p. 3958-3967.

[5] H. Hecht, "Prognostics for electronic equipment: an economic perspective," Proceedings RAMS Conference, Newport Beach, CA; January 2006, p. 165-168.

[6] G. Vachtsevanos, "Cost and complexity trade-offs in prognostics," Proceedings NDIA Intelligent Vehicles Systems Symposium, Traverse City, MI; June 2003.

[7] J.E. Land, "HUMS-the benefits-past, present and future," Proceedings of IEEE Aerospace Conference, Big Sky, MT; March 2001, Vol. 6, p.3083-3094.

[8] B. Larder, "Assessing the benefit of helicopter health and usage monitoring systems," IEE Seminar on Aircraft Airborne Condition Monitoring; 14 May 2003, p. 1-3

[9] J.M. Poradier, M. Trouve, "An assessment of Eurocopter experience in HUMS development and support," 57 $7^{\text {th }}$ AHS International Annual Forum, Washington, DC; May 2001.

[10] K. Pipe, "Measuring the performance of a HUM system - features that count," 3rd DSTO International Conference on Health and Usage Monitoring, Melbourne; February 2003.

[11] Operability in Systems Concept and Design: Survey, Assessment, and Implementation, Uwohali, Inc, 1996. Available at: 
Microelectronics Reliability, Vol. 47, No. 12, pp. 1857-1864, December 2007

http://science.ksc.nasa.gov/shuttle/nexgen/Nexgen_Downloads/Ops_Survey_of_Tools_Report_by_MSFC_ 1996.pdf

[12] P. Sandborn, C. Wilkinson, "A maintenance planning and business case development model for determining the applicability of prognostic health management (PHM) approaches to electronic systems," Microelectronic Reliability, Special Issue on Electronic Prognostics and Health Management; 15 October 2007.

[13] J.B. Bernstein, "Early end of life failures of electronics in avionic systems", AVSI Project No. 17: Methods to account for accelerated semiconductor device wear-out; May 2001.

[14] L. Condra, "Integrated aerospace parts acquisition strategy," Technical committee GEL/107. Process management for Avionics, BSI Chiswick; 7 October 2002.

[15] L. V. Kirkland, T. Pombo, K. Nelson, F. Berghout, “Avionics health management: searching for the prognostics grail," Proceedings of IEEE Aerospace Conference, Big Sky, MT; March 6-13, 2004, Vol. 5, p. $3448-3454$.

[16] A. Ramakrishnan, M. Pecht, "A Life Consumption Monitoring Methodology for Electronic Systems," IEEE Transactions on Components and Packaging Technologies, (September) 2003, pp. 625-634.

[17] R. Gopalan, K.T. Talluri, "The aircraft maintenance routing problem," Operations Research; 1998; Vol. 46, No. 2, p. 260-271. 